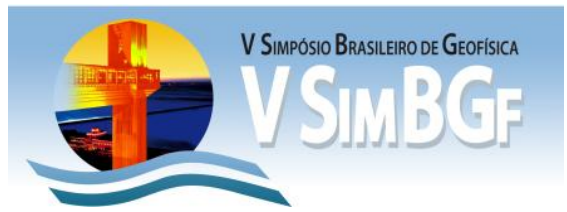

\title{
ANOMALIA MAGNÉTICA DO ATLÂNTICO SUL NOS ÚLTIMOS 3000 ANOS
}

Filipe S. Terra-Nova, Gelvam A. Hartmann, Ricardo I.F. Trindade, Igor I.G. Pacca

Universidade de São Paulo, São Paulo, Brasil

Instituto de Astronomia, Geofísica e Ciências Atmosféricas

Departamento de Geofísica

\section{Resumo}

O campo magnético da Terra (CMT) apresenta feições que são persistentes no tempo. A Anomalia Magnética do Atlântico Sul (SAMA) é a mais importante delas e apresenta variações que podem ser descritas através dos modelos do campo geomagnético. Neste trabalho foi utilizado o modelo CALS3k.4 para descrever as principais características da SAMA nos últimos 3000 anos. Os resultados indicam que a SAMA apresenta deriva para Oeste e Leste em diferentes intervalos de tempo. As variações de intensidade são devidas principalmente pelas oscilações das componentes quadrupolar e octopolar do campo.

\section{Introdução}

O campo magnético da Terra (CMT) apresenta variações tanto espaciais como temporais, tendo como fontes o campo interno (gerado no núcleo externo da Terra), campo externo (magnetosfera) e o campo de origem crustal. Cada uma dessas fontes pode ser distinguida de medidas do campo através de modelos globais do campo geomagnético desenvolvidos pela análise por harmônicos esféricos. Este tipo de análise, que é a mais usual para descrição do CMT, permite separar as diferentes componentes do campo, que são a componente dipolar e as componentes não-dipolares (e.g. Merrill et al, 1998).

Dados de magnetismo fóssil registrado em materiais geológicos e arqueológicos revelam que nos últimos milênios o CMT é dominantemente dipolar e que nos últimos séculos o momento de dipolo está diminuindo continuamente (e.g. Bloxham et al., 1989; Hulot et al., 2010). Essa queda no momento de dipolo está associada à presença de uma feição não-dipolar de grande escala situada no hemisfério Sul, a Anomalia Magnética do Atlântico Sul (SAMA, em inglês) (Olson e Amit, 2006). Essa anomalia apresenta os mais baixos valores de intensidade do campo observados na superfície da Terra para faixas de mesma latitude. A origem da SAMA está intrinsicamente relacionada aos movimentos do fluido condutor no núcleo externo da Terra (Olson e Amit, 2006).

A evolução temporal da SAMA pode ser descrita pelos modelos de campo por harmônicos esféricos devido à possibilidade de separação das diferentes componentes do campo. Hartmann e Pacca (2009), usando o modelo GUFM1 (Jackson et al., 2000), descreveram a deriva para o oeste da SAMA e indicaram as possíveis causas da queda da intensidade total, relacionando esse fato à forte presença dos campos quadrupolar e octopolar. No entanto, sabe-se que modelos mais recentes indicam diferentes variações para o coeficiente que descreve o campo dipolar (Gubbins et al., 2006; Finlay, 2008), bem como modelos de alta resolução que avançam no tempo (Korte et al., 2011). Dessa forma, grandes feições do campo como a SAMA podem apresentar diferentes tendências de variação devido aos diferentes modelos de campo. Neste trabalho são avaliadas essas diferenças utilizando o modelo de campo CALS3k.4 (Korte et al., 2011) para o intervalo de 1000 AC até 2000 AD. Serão abordados os aspectos de deriva para Oeste, variação de intensidade total calculado na superfície da Terra e variações das componentes não-dipolares.

\section{Metodologia}

\section{Modelagem e modelos do campo geomagnético}

O CMT é um campo vetorial representado por B (indução magnética) sendo mais comumente utilizado do que $\mathrm{H}$ (intensidade magnética). $O$ campo $B$ deve respeitar as leis de Maxwell. Tomando as equações de Maxwell e admitindo ausência de magnetização, o CMT é descrito por:

$$
\nabla^{2} V=0 \text { (Equação de Laplace) }
$$

A equação diferencial parcial (EDP) é solucionada pelo método de separação de variáveis, obtendo-se como solução:

$$
V=\sum_{n=0}^{\infty} \sum_{m=0}^{n}\left[C_{n}^{m} r^{n}+D_{n}^{m} r^{-(n+1)}\right] P_{n}^{m}\left[A_{n}^{m} \cos m \lambda+B_{n}^{m} \operatorname{senm} \lambda\right]
$$

A solução para a variável $\theta$ são os Polinômios de Legendre $\left(P_{n}^{m}\right)$, variando em ordem $\mathrm{n}$ e grau $\mathrm{m}$. A partir desta equação e relacionando à variável $r$ com o raio da Terra a chega-se a uma expressão onde os coeficientes e são chamados coeficientes de Gauss, que compõe um modelo de campo. A expressão fica:

$V=a \sum_{n=0}^{\infty} \sum_{m=0}^{n}\left[\left(\frac{a}{r}\right)^{(n+1)}\right] P_{n}^{m}\left[g_{n}^{m} \cos m \lambda+h_{n}^{m} \operatorname{sen} m \lambda\right]$

O potencial $V$ não é diretamente observado na superfície. Os dados obtidos através de medidas (componentes $\mathrm{X}, \mathrm{Y}$ 
e $\mathbf{Z}$ do campo geomagnético) podem ser relacionados ao potencial através das seguintes expressões:

$$
\begin{aligned}
& X=\sum_{n=1}^{\infty} \sum_{m=0}^{n} \frac{d P_{n}^{m}}{d \theta}\left[g_{n}^{m} \cos m \lambda+h_{n}^{m} \operatorname{sen} m \lambda\right] \\
& Y=\frac{1}{\operatorname{sen} \theta} \sum_{n=1}^{\infty} \sum_{m=0}^{n} P_{n}^{m}\left[m g_{n}^{m} \operatorname{sen} m \lambda-m h_{n}^{m} \cos m \lambda\right] \\
& Z=\sum_{n=1}^{\infty} \sum_{m=0}^{n} P_{n}^{m}\left\{\left[n c_{n}^{m}-(n+1)\left(1-c_{n}^{m}\right)\right] g_{n}^{m} \cos m \lambda\right. \\
& \left.+\left[{ }^{m} S_{n}^{m}-(n+1)\left(1-S_{n}^{m}\right) h_{n}^{m} \operatorname{sen} m \lambda\right]\right\}
\end{aligned}
$$

Com os vários valores das componentes do campo espalhados em diversos pontos da Terra determinam-se os coeficientes de Gauss.

Modelos arqueomagnéticos para os últimos 3.000 anos (Korte et al., 2011)

Korte et al. (2011) desenvolveram um modelo para os últimos 3000 anos utilizando diferentes bases de dados compilados por Korhonen et al. (2008), Genevey et al. (2008) e Donadini et al. (2009), dentre outras fontes de dados mais recentes. É importante notar que o período de 3000 anos foi escolhido porque apresenta a maior concentração de dados de direção e intensidade. O modelo CALS3k.4 incorpora duas bases de dados, que são estes dados arqueomagnéticos e dados de sedimentos de lagos. O modelo CALS3k.4 tem seus coeficientes de Gauss oriundos da incorporação de dados geomagnéticos e arqueomagnéticos. Tais coeficientes implicam diretamente na interpretação das principais feições do CMT, como por exemplo, a SAMA. Portanto, a evolução temporal dessas feições pode ser estudada utilizando esse modelo de campo.

\section{Resultados}

A metodologia computacional desenvolvida foi validada através do cálculo do campo utilizando os modelos do IGRF (International Geomagnetic Reference Field) e GUFM1 (Jackson, et al, 2000). Os resultados obtidos com o modelo CALS3k.4 foram comparados com aqueles previamente obtidos em Hartmann e Pacca (2009), mostrando que a metodologia computacional é compatível e adequada para a utilização em modelos construídos a partir de dados arqueomagnéticos.

A Figura 1 mostra os resultados de intensidade total determinada pelo modelo CALS3k.4. A evolução temporal do campo total indica que as principais feições do CMT são descritas pelo modelo CALS3k.4. Outros núcleos de baixa intensidade diferentes da SAMA aparecem em tempos inferiores à 1100 anos. Os maiores valores de campo total são verificados no ano 0000 .
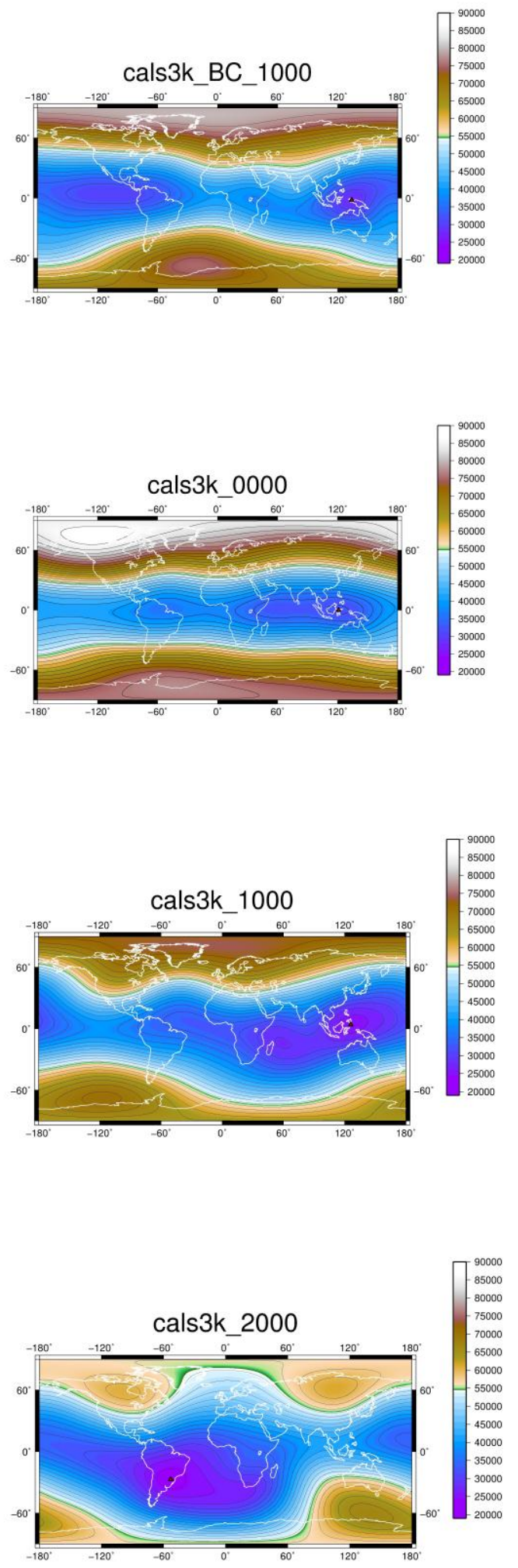

Figura 1: Resultados do campo total. Os resultados foram calculados para os anos 1000 AC, 0000, 1000 AD, 2000 
$A D$. O triângulo vermelho indica o ponto de menor intensidade do campo total;

Na figura 2 é descrita a razão não dipolar pros últimos 3000 anos. Os pontos onde a razão é máxima aproximam-se dos pontos onde a intensidade é mínima na descrição do campo total (Figura 1).
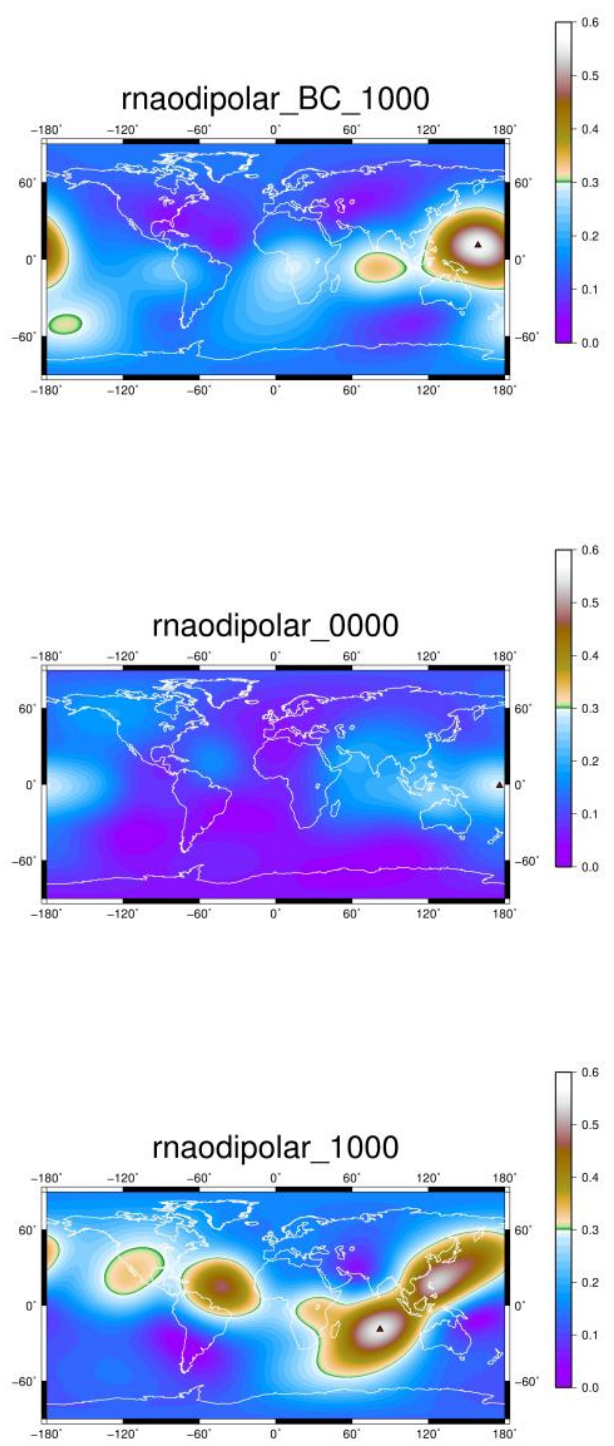

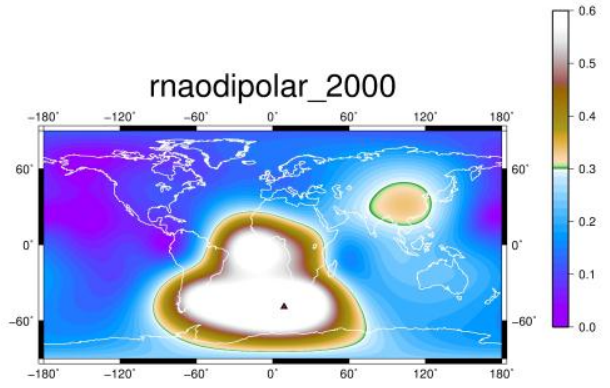

Figura 2: Resultados da razão campo não dipolar por campo total. Os resultados foram calculados para os anos $1000 A C, 0000,1000 A D, 2000 A D$. O triângulo vermelho indica o ponto de maior razão.

A figura 3 descreve a razão quadrupolar pros últimos 3000 anos. Os pontos onde a razão é máxima aproximam-se novamente dos pontos onde a intensidade é mínima na descrição do campo total, As feições descritas pelas diferentes razões são próximas visualmente, mas apresentam diferenças na intensidade.
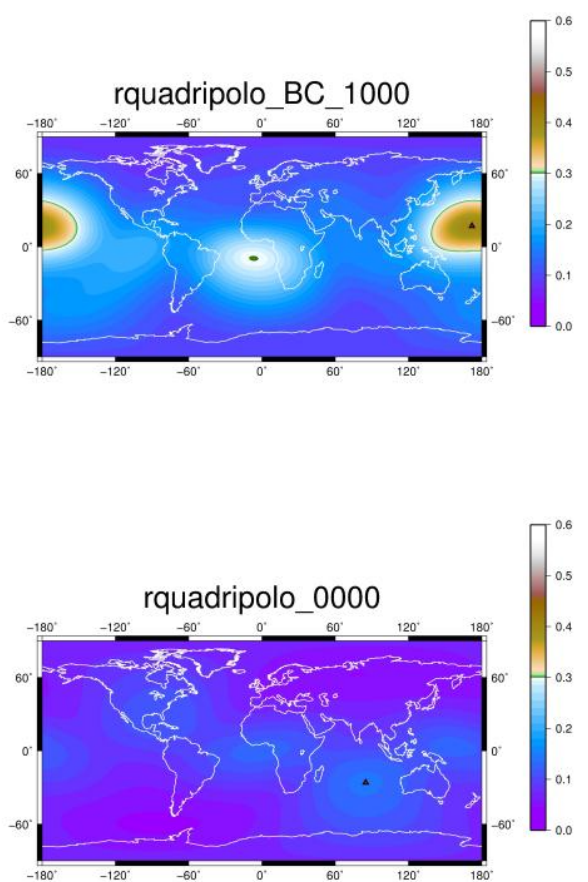


\section{Referências}
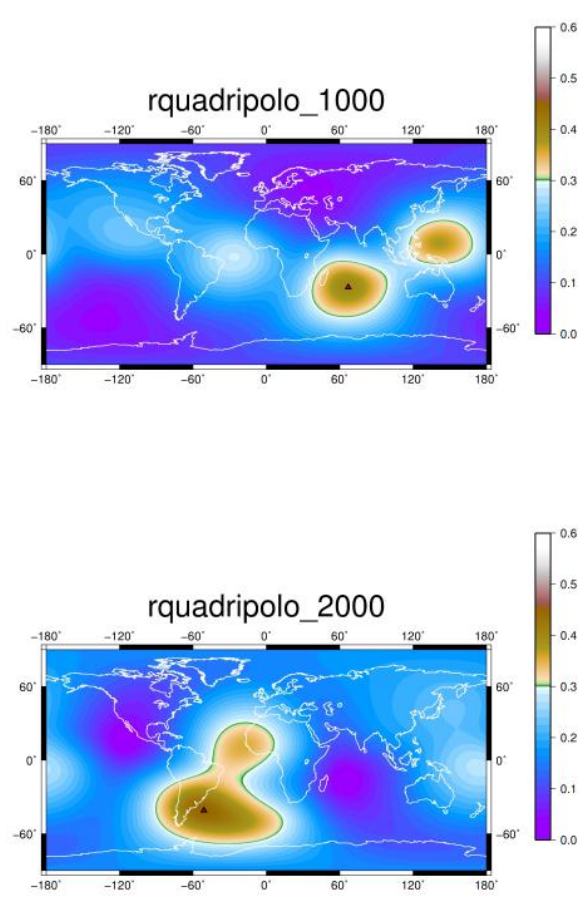

Figura 3: Resultados da razão campo quadrupolar por campo total. Os resultados foram calculados para os anos $1000 A C, 0000,1000 A D, 2000 A D$. O triângulo vermelho indica o ponto de maior razão.

\section{Discussão}

Como visto em Hartmann e Pacca (2009), a SAMA é influenciada pelo campo não-dipolar e consequentemente pelo campo quadrupolar. No entanto foi verificado que a deriva para o Oeste nem sempre ocorre, sendo que a direção da deriva da SAMA altera-se com o passar do tempo.

Outro resultado importante consta que a SAMA nem sempre existiu. Outras anomalias aparecem ao se recuar no tempo, de maneira que não representam um deslocamento da SAMA e sim uma mudança drástica de posição do campo de menor intensidade do CMT.

\section{Agradecimentos}

Agradecemos ao apoio do Instituto de Astronomia Geofísica e Ciências Atmosféricas, e mais especificamente ao Departamento de Geofísica, e ao Banco Santander por financiar a nossa pesquisa.
Bloxham, J., Gubbins, D., Jackson, A., 1989. Geomagnetic Secular Variation. Phil. Trans. Royal Soc. London A, 329, 415-502.

Donadini, F., Korte, M., Constable, C.G., 2009. Geomagnetic field for $0-3 \mathrm{ka}$ : 1 . New data sets for global modeling. Geochem. Geophys. Geosyst., 10, 6, Q06007.

Finlay, C.C., 2008. Historical variation of the geomagnetic axial dipole. Phys. Earth Planet. Int., 170, 1-14.

Genevey, A., Gallet, Y., Rosen, J., Le Goff, M., 2009. Evidence for rapid geomagnetic field intensity variations in Western Europe over the past 800 years from new French archeomagnetic data. Earth Planet. Sci. Lett., 284, 132-143.

Gubbins, D., Jones, A.L., Finlay, C.C., 2006. Fall in Earth's Magnetic Field is Erratic. Science, 312, 900-902.

Hartmann, G.A., Pacca, I.G., 2009. Time evolution of the South Atlantic Magnetic Anomaly. An. Acad. Bras. Ciênc., 81, 243-255.

Hulot, G., Finlay, C.C., Constable, C.G., Olsen, N., Mandea, M., 2010. The magnetic field of the planet Earth. Space Sci. Rev., 152,159-222.

Jackson, A., Jonkers, A.R.T., Walker, M., 2000. Four centuries of geomagnetic secular variation from historical records. Phil. Trans. R. Soc. London, A 358, 957-990.

Korhonen, K., Donadini, F., Riisager, P., Pesonen, L.J., 2008. GEOMAGIA50: An archeointensity database with PHP and MySQL. Geochem. Geophys. Geosyst., 9, Q04029.

Korte, M., Constable, C.G., 2011. Improving Geomagnetic field Reconstructions for $0-3 k a$. Physics of the Earth and Planetary Interiors Vol. 188, Issue 3-4, October 2011, Pages 247-259.

Merrill, R.T., McElhinny, M.W., McFadden, P.L., 1998. The magnetic field of the Earth: Paleomagnetism, the core, and the deep mantle, Vol. 63 (International Geophysics Series.), Elsevier, Academic Press, Amsterdam.

Olson, P., 2002. The disappearing dipole. Nature, 416, 591-594.

Olson, P., Amit, H., 2006. Changes in Earth's dipole, Naturwissenschaften, 93, 519-542. 